\title{
Fungal Infections in the Elderly
}

\author{
Angela Revelas ${ }^{1}$, Emmanuel Liannos ${ }^{2}$, Dimitrios Arvanitakis ${ }^{3}$ \\ ${ }^{I}$ Pathological Department of St.Nicolaos Hospital -Crete, Greece. \\ ${ }^{2}$ Urological Department of St.Nicolaos Hospital-Crete, Greece. \\ ${ }^{3}$ Neurosurgery Clinic of Venizeleio General Hospital-Crete, Greece.
}

Corresponding

Author:Angela Revelas

E.mail:donnoiko@gma il.com

Received : $11 / 12$

12012

Accepted after

revision: 5 /2/2013

Key words:

Elderly, infections,

candidiasis, skin, illness, drugs.
Fungi are increasingly recognised as major pathogens in critically ill patients. Several reasons have been proposed for the increase in invasive fungal infections, including the use of antineoplastic and immunosuppressive agents, broadspectrum antibiotics, and prosthetic devices and grafts, and more aggressive surgery. Patients with burns, neutropenia, HIV infection and pancreatitis are also predisposed to fungal infection Candidiasis is caused by infection with species of the genus Candida, predominantly with Candida albicans.
Candida species are ubiquitous fungi that represent the most common fungal pathogens that affect humans. The growing problem of mucosal and systemic candidiasis reflects the enormous increase in the number of patients at risk and the increased opportunity that exists for Candida species to invade tissues normally resistant to invasion.

\section{INTRODUCTION}

Candida species are true opportunistic pathogens that exploit recent technological advances to gain access to the circulation and deep tissues. The increased prevalence of local and systemic disease caused by Candida species has resulted in numerous new clinical syndromes, the expression of which depends primarily on the immune status of the host. Candida species produce a wide spectrum of diseases, ranging from superficial mucocutaneous disease to invasive illnesses, such as hepatosplenic candidiasis, Candida peritonitis, and systemic candidiasis.

The management of serious and lifethreatening invasive candidiasis remains severely hampered by delays in diagnosis and the lack of reliable diagnostic methods that allow detection of both fungemia and tissue invasion by Candida species.

Advances in medical technology, chemotherapeutics, cancer therapy, and organ transplantation have greatly reduced the morbidity and mortality of life-threatening disease. Patients who are critically ill and in medical and surgical ICUs have been the prime targets for opportunistic nosocomial fungal infections, primarily due to Candida species. Studies suggest that the problem is not under control and, in fact, show it is worsening. On a daily basis, virtually all physicians are confronted with a positive Candida isolate obtained from one or more various anatomical sites. High-risk areas for Candida infection include neonatal, pediatric, and adult ICUs, both medical and surgical[1]. Candida infections can involve any anatomical structure.

\section{Candida}

Over 200 species of Candida exist in nature; thus far, only a few species have been associated with disease in humans.

The medically significant Candida species include the following[2]:

$C$ albicans, the most common species identified (50-60\%)

Candida glabrata (previously known as Torulopsis glabrata) (15-20\%)

C parapsilosis (10-20\%)

Candida tropicalis (6-12\%)

Candida krusei (1-3\%) 
Candida kefyr $(<5 \%)$

Candida guilliermondi $(<5 \%)$

Candida lusitaniae $(<5 \%)$

Candida dubliniensis, primarily recovered from patients infected with infected with HIV

$C$ glabrata and $C$ albicans account for approximately $70-80 \%$ of Candida species recovered from patients with candidemia or invasive candidiasis. $C$ glabrata has recently become very important because of its increasing incidence worldwide, its association with fluconazole resistance in up to $20 \%$ of clinical specimens, and its overall decreased susceptibility to other azoles and polyenes.

C krusei is important because of its intrinsic resistance to ketoconazole and fluconazole (Diflucan); it is also less susceptible to all other antifungals, including itraconazole (Sporanox) and amphotericin B.

Another important Candida species is $C$ lusitaniae; although not as common as other Candida species, $C$ lusitaniae is of clinical significance because it may be intrinsically resistant to amphotericin $\mathrm{B}$, although it remains susceptible to azoles and echinocandins.

$C$ parapsilosis is also an important species to consider in hospitalized patients. It is especially common in infections associated with vascular catheters prosthetic devices. Additionally, in vitro analyses have shown that echinocandins have a higher minimum inhibitory concentration (MIC) against $C$ parapsilosis than other Candida species. The clinical relevance of this in vitro finding has yet to be determined [3].

$C$ tropicalis has frequently been considered an important cause of candidemia in patients with cancer (leukemia) and in those who have undergone bone marrow transplantation.

\section{Oral candidiasis.}

White plaques that are present on the buccal, palatal, or oropharyngeal mucosa and can easily be removed are the typical lesions seen in patients with oral candidiasis.Some patients develop painful cracks at the corns of the mouth [angular cheilitis].Factors that predispose patients to the development of thrush include xerostomia,the use of broad-spectrum antibiotics, inhaled corticosteroids, and diminished cellmediated immunity. Age alone is not sufficient for the development of oral candidiasis .In an older adult, oral thrush-in the absence of an obvious cause or extension to involve the esophagus-may herald underlying immunosuppression in the form of cancer or AIDS. The presence of xerostomia has been shown to correlate with both increased colonization of the oropharynx and increase oral mucosal lesions due to yeast [4]. In older adults, the presence of systemic diseases and a multiplicity of medications can frequently cause xerostomia, which may than place patients at risk of developing oral thrush.

\section{Denture stomatitis.}

Denture stomatitis, a variant of oral candidiasis, presents as chronic mucosal erythema beneath a denture. This may affect up to $65 \%$ of all patients who wear dentures, occurring particularly in those with full sets[5].Patients who do not remove the dentures at night and who have poor oral hygiene are the most likely to be affected.

\section{Skin and nail infections.}

Candidal infection of the skin under the breasts, a pannus,or the perineum occurs when these areas become macerated. Lesions are almost always pruritic, erythematous, and have a distinct border. Small, flat, erythematous satellite lesions provide an additional clue to the diagnosis of candidiasis.

\section{Paronychia and onychomycosis.}

Candida uncommonly causes infection in the periungual area and underneath the nailbed. Inflammation leads to thickening and even loss of the nail. The disease occurs most often in persons who frequently immerse their hands in water and has not been a major problem in older patients. However, one group of older adults in whom onychomycosis can have serious consequences in those with diabetes mellitus. In comparison with nondiabetic patients, patients with diabetes have both increase susceptibility to and worse outcome from bacterial infection of the feet. Onychomycosis can contribute to difficulty cutting the toenails, predisposing these patient to trauma and thus bacterial foot infection. Onychomycosis is found more frequently in the elderly and more often in males than females. There are four types of onychomycosis; distal subungual onychomycosis, proximal subungual onychomycosis, white superficial onychomycosis, and candidal onychomycosis. 


\section{Vulvovaginal candidiasis (VVC)}

This is the second most common cause of vaginitis. The patient's history includes vulvar pruritus, vaginal discharge, dysuria, and dyspareunia. Approximately $10 \%$ of women experience repeated attacks of VVC without precipitating risk factors. Physical examination findings include a vagina and labia that are usually erythematous, a thick curdlike discharge, and a normal cervix upon speculum examination [6].

Candiduria often presents a dilemma to the clinician as it may represent contamination, colonization,or infection. Contamination may be detected by repeating collection and culture of the urine. However, straight catheterization of the urethra may be needed to obtain an uncontaminated urinary specimen in older women. In the asymptomatic patient,some clinicians consider the presence of any yeast in the urine to represent infection, whereas some authors suggest that $<10,000 \mathrm{cfu} / \mathrm{mL}$ may represent only colonization[7]. The presence of a urinary catheter limits the usefulness of colony quantitation. Most authors would agree that the presence of symptoms such as suprapubic discomfort, dysuria, or frequency usually comprise infection, rather than colonization. Asymptomatic candiduria: Most catheterized patients with persistent candiduria are asymptomatic, similar to noncatheterized patients. Most patients with candiduria have easily identifiable risk factors for Candida colonization. Thus, invasive disease is difficult to differentiate from colonization based solely on culture results because approximately $5-10 \%$ of all urine cultures are positive for Candida [8].

\section{Candidemia}

Candida species are currently the fourth most commonly isolated organism in blood cultures, and Candida infection is generally considered a nosocomial infection [9]. The patient's history commonly reveals the following:

Several days of fever that is unresponsive to broad-spectrum antimicrobials; frequently the only marker of infection

Prolonged intravenous catheterization

A history of several key risk factors

Possibly associated with multiorgan infection
Physical examination results may include the following:

-Fever.

-Macronodular skin lesions (approximately $10 \%)$.

-Candidal endophthalmitis (approximately 10$28 \%$ ).

-Occasionally, septic shock (hypotension, tachycardia, tachypnea).

\section{CONCLUSION}

Infections in the elderly are more common than in younger individuals. They are more complicated by the multiple medications used to control the diseases that accompany normal ageing. Understanding the diseases an elderly patient might suffer enhances their health and well being.

\section{REFERENCES}

1. Pappas PG, Rex JH, Lee J, Hamill RJ, Larsen RA, Powderly W, et al. A prospective observational study of candidemia: epidemiology, therapy, and influences on mortality in hospitalized adult and pediatric patients. Clin Infect Dis 2003;37(5):634-43.

2. Vazquez JA, Sobel JD. Candidiasis. In: Clinical Mycology, Dismukes WE, Pappas PG, and Sobel JD, eds. Oxford Univers 2003:143-87

3. Eiland EH, Hassoun A, English T. Points of concern related to the micafungin versus caspofungin trial. Clin Infect Dis 2008;46(4):640-1; author reply 641

4. Narhi TO, Almano A, Meurman JH. Salivary yeasts, saliva and oral mucosa in the elderly.J.Dent.Res 1993;72:1009-14.

5. Budtz-Jorgensen E. Oral mucosal lesions associated with the wearing of removable dentures.J.Oral.Pathol 1981;10:65-80.

6. Sobel JD. Vulvovaginal candidosis. Lancet 2007;369 (9577):1961-71.

7. Jacobs LG, Skidmore EA, Freeman K, Lipschultz D, Fox N. Oral fluconazole compared with bladder irrigation with amphotericin $\mathrm{B}$ for treatment of fungal urinary tract infections in elderly patients. Clin. Infect. Dis 1996;22:30-5.

8. Malani AN, Kauffman CA. Candida urinary tract infections: treatment options. Expert Rev Anti Infect Ther 2007;5(2):277-84. 
9. Guery BP, Arendrup MC, Auzinger G, Azoulay E, Borges Sá M, Johnson EM, et al. Management of invasive candidiasis and candidemia in adult non-neutropenic intensive care unit patients: Part I. Epidemiology and diagnosis. Intensive Care Med 2009;35(1):55-62 